Magalhães, C., Paço, A., Alonso, H., \& Oliveira, M. (2021). Marketing Verde: Comparando o consumo de produtos ecológicos nas gerações X e Y. Consumer Behavior Review, 5(3), 339-355.

DOI: https://doi.org/10.51359/2526-7884.2021.250619

ISSN: 2526-7884

Editor: Prof. Dr. Marconi Freitas da Costa

E-mail: cbr@ufpe.br
Avaliação: Double blind review Recebido: 25 de maio de 2021 Aprovado: 15 de julho de 2021

\title{
MARKETING VERDE: COMPARANDO O CONSUMO DE PRODUTOS ECOLÓGICOS NAS GERAÇÕES X E Y
}

Green marketing: comparing the consumption of ecological products of generations $X$ and $Y$

\author{
Carla Magalhães ${ }^{1}$ \\ ORCID: http://orcid.org/0000-0002-0193-971X \\ E-mail: carla.magalhaes@ulp.pt \\ Arminda Paço ${ }^{2}$ \\ ORCID: http://orcid.org/0000-0002-2806-4247 \\ E-mail: apaco@ubi.pt \\ Hugo Alonso ${ }^{1,3}$ \\ ORCID: http://orcid.org/0000-0002-1599-5392 \\ E-mail: hugo.alonso@ulp.pt \\ Marta Oliveira ${ }^{1}$ \\ ORCID: https://orcid.org/0000-0002-1633-7930 \\ E-mail: santosoliveiramarta@gmail.com \\ ${ }^{1}$ Universidade Lusófona do Porto, TRIE, Porto, Portugal \\ ${ }^{2}$ Universidade da Beira Interior, Covilhã, Portugal \\ ${ }^{3}$ Universidade de Aveiro, CIDMA, Aveiro, Portugal
}

\section{Resumo}

Este estudo analisa a influência de determinados estímulos de marketing (propaganda, informação veiculada e preço) no consumo de produtos ecológicos,

\section{Abstract}

This study analyzes the influence of certain marketing stimuli (advertising, information conveyed and price) on the consumption of ecological products, comparing the behavior 
comparando o comportamento dos consumidores portugueses das gerações $\mathrm{X}$ e $\mathrm{Y}$. Através de uma pesquisa quantitativa, crosssectional, com base num questionário online, cujos resultados foram analisados com recurso ao software SPSS Statistics 25, concluímos que existem algumas semelhanças entre ambas as gerações, como a capacidade de identificação dos produtos ecológicos e a predisposição para a sua compra, o impacto positivo das campanhas de comunicação com apelo emocional e a perceção da importância dos rótulos dos produtos ecológicos. A variável que mais distingue o comportamento de ambas as gerações é o preço, pois a geração Y está mais predisposta a pagar um valor superior por um produto ecológico. Esta investigação contribui para a literatura sobre o comportamento do consumidor, especialmente no âmbito da variável "geração", aplicado ao contexto do consumo de produtos ecológicos. Também ajuda as empresas a posicionarem-se melhor na relação com os consumidores de ambas as gerações analisadas. A definição de estratégias de targeting mais acuradas relativamente à promoção, preço e decisão de compra pode então tomar como ponto de partida os resultados deste estudo.

Palavras-chave: Marketing verde; Produtos ecológicos; Estímulos de marketing; Geração X; Geração Y. of Portuguese consumers of generations $\mathrm{X}$ and Y. Through a quantitative, cross-sectional research, based on an online questionnaire, whose results were analyzed using the SPSS Statistics 25 software, we concluded that there are some similarities between both generations, such as the ability to identify ecological products and the predisposition for their purchase, the positive impact of communication campaigns with emotional appeal and the perception of the importance of the labels of ecological products. The variable that most distinguishes the behavior of both generations is price, since generation $\mathrm{Y}$ is more likely to pay a higher price for an ecological product. This investigation contributes to the literature on consumer behavior, especially within the scope of the "generation" variable, applied to the context of consumption of ecological products. It also helps companies to better position themselves in the relationship with consumers of both generations analyzed. The definition of more accurate targeting strategies in relation to promotion, price and purchase decision can then take as a starting point the results of this study.

Keywords: green marketing; ecological products; marketing stimuli; generation $\mathrm{X}$; generation $\mathrm{Y}$.

\section{INTRODUÇÃO}

O marketing verde utiliza os princípios do marketing tradicional, porém, é orientado para o segmento ecológico. Visa a satisfação das necessidades dos consumidores e da sociedade, de uma forma rentável e sustentável e, em simultâneo, compatível com o meio ambiente e os eco-sistemas (Papadas, Avlonitis \& Carrigan, 2017). Existem algumas ferramentas especialmente importantes neste âmbito por facilitarem a identificação dos atributos ecológicos dos produtos, nomeadamente os rótulos, a propaganda e a associação à marca (Rahbar \& Wahid, 2011). Por sua vez, os consumidores cumprem um papel de relevo no mercado verde, podendo contribuir para o desenvolvimento sustentável e ajudar a prevenir ou diminuir os impactos ambientais negativos através da compra de produtos ecológicos (Joshi \& Rahman, 2016).

No mercado consumidor, existe a geração X que é composta por consumidores mais velhos, sendo um segmento importante e relevante no mercado atual, que tem vindo a vivenciar muitas mudanças sociais, culturais e tecnológicas (Mitchell, McLean \& Turner, 2005). Esta geração coexiste com a geração Y, também designada como a geração dos millennials, conhecida por ser "revolucionadora", tendo sido pioneira na utilização das redes sociais e plataformas digitais (Jang, Kim \& Bonn, 2011). Enquanto consumidores são também distintos: se, por um lado, temos a geração $X$ caracterizada por ser sensível ao preço, curiosa em experimentar novos produtos e pragmática nas suas escolhas (Williams \& Page, 2011), por outro lado, temos os consumidores da geração Y, para os 
quais a influência social é uma das maiores fontes de preocupação e motivação para determinadas compras e as marcas desempenham um papel de relevo (Parment, 2013) e que, sendo sensíveis ao preço, são propensos a comprar produtos dispendiosos se os considerarem de prestígio (Williams \& Page, 2011). Aliás, estímulos como o impacto social, têm vindo a ser estudados por outros autores, comprovando que a experiência de consumo está muito associada a fatores sociais (Farias \& Aguiar, 2015) e culturais (Côrtes \& Moretti, 2013).

Parecem existir já inúmeros estudos focados no comportamento do consumidor numa ou noutra geração; no entanto, são poucos os que incidem na análise das diferenças entre gerações no que respeita ao consumo de produtos amigos do ambiente, e nas variáveis que influenciam esse mesmo comportamento. Este estudo visa precisamente colmatar esta lacuna e contribuir para o desenho de implicações práticas para as organizações melhor atenderem às diferenças intergeracionais entre os consumidores, nomeadamente, perceber de que forma as empresas podem ajustar variáveis como o preço, propaganda, comunicação e informação, e rotulagem, entre outras, para melhor irem ao encontro, ora das necessidades e anseios de uma geração, ora dos desejos da outra.

Este estudo foca-se na comparação dos comportamentos das duas gerações, a X e a $Y$, no que respeita à sua resposta aos diferentes estímulos do marketing verde. Para tal, ir-se-á descrever o consumidor verde, caraterizando as gerações em análise e abordando alguns estímulos de marketing que poderão influenciar o comportamento desses consumidores. Posteriormente, será apresentada a metodologia do estudo, os resultados e respetiva discussão.

\section{REVISÃO DA LITERATURA $O$ consumidor verde}

Shrum, McCarty e Lowrey (1995) definiram o consumidor verde como qualquer pessoa cujo comportamento de compra é influenciado por preocupações ambientais. 0 estudo conduzido por estes autores conclui que estes consumidores se consideram líderes de opinião, mostram interesse em novos produtos, trocam informações sobre produtos de forma ativa e são cuidadosos nos seus hábitos de consumo, mostrando sempre sensibilidade ao preço. 0 esforço de comprar produtos ecológicos não está relacionado com compras de impulso e com a lealdade à marca.

O comportamento ecológico consciente e a intenção de compra ecológica são elementos preditivos do comportamento de compra, embora a maior influência neste comportamento advenha do comportamento ecológico consciente. As variáveis psicográficas (personalidade, motivações e estilo de vida) são mais relevantes na explicação do comportamento ecológico do que as variáveis sociodemográficas (idade, gênero, escolaridade e classe social), nomeadamente o sentimento de altruísmo e a percebida eficácia do comportamento do consumidor (Akehurst, Afonso \& Gonçalves, 2012). Existe, contudo, uma relação positiva entre o nível de escolarização e a consciencialização ambiental/comportamentos ecológicos.

0 consumidor verde também dá importância à qualidade e ao preço das marcas que adotam práticas de preservação e preocupação ambiental (Ciribele \& Caneschi, 2011). Sousa, Castro e Oliveira (2016), também indicam que os consumidores verdes poderiam investir mais no fator preço, se os locais onde compram os seus produtos tivessem uma política mais ecológica. Porém, esta questão não consensual, já que o preço não deixa de ser um fator sensível aos consumidores, mesmo aos que têm um perfil mais ecológico, o que pode interferir negativamente com a compra (Kianpour, Jusoh \& Asghari, 2012).

O estudo de Pickett-Baker e Ozaki (2008), conclui que o comportamento ecológico não é significativamente influenciado pelas convicções ecológicas. Encontra-se, portanto, uma lacuna entre as convicções ambientais e os comportamentos ecológicos. Existe uma maior probabilidade dos valores pró-ambientais resultarem em comportamentos pró-ambientais quando os valores são suficientemente específicos e as ações ecológicas tomadas pelos consumidores irão de alguma forma ao encontro dos seus interesses. Este processo é também influenciado pelos atributos dos produtos quando estes são percebidos positivamente (Pickett-Baker \& Ozaki, 2008).

A relação entre a atitude e o comportamento pode ser fortalecida através do aumento da consciência ecológica, da disponibilidade de pagar um preço premium, dos benefícios para a saúde 
pessoal e para a comunidade, da motivação percebida da organização, da disponibilidade dos produtos, da vontade de passar tempo adicional na compra de produtos ecológicos, e ainda, dos esforços de comunicação das organizações (Goriparthi \& Tallapaly, 2017).

Um dos pontos críticos para o marketing verde é o reduzido benefício percebido pela maioria dos consumidores na compra deste tipo de produtos. Este desafio pode ser superado pela associação de valor emocional às marcas ecológicas com o objetivo de aumentar o benefício individual percebido (Hartmann \& Ibáñez, 2006). Também é importante perceber que fatores os consumidores valorizam, em termos ambientais, pois isso ajuda-nos a perceber o que é realmente, na prática, um consumidor ecológico (Júnior et al., 2020).

A identificação de produtos ecológicos difere consoante a preocupação ambiental dos consumidores. Um dos motivos pelo qual os consumidores não consideram os produtos ecológicos na sua decisão de compra é a falta de informação sobre os mesmos, pelo que devem ser feitos esforços de marketing de forma de combater esta falta de informação e esclarecer os consumidores sobre os produtos ecológicos e as suas vantagens (Motta, 2008).

De resto, outros fatores também poderão influenciar o consumo ecológico, tais como os sentimentos do consumidor (Lima, Costa \& Félix, 2019). Também é preciso salientar que nem sempre as estratégias de Marketing Verde utilizadas pelas empresas conduzem a um sentimento de confiança por parte dos consumidores (Mangini, Amaral, Conejero \& Pires, 2020).

\section{A geração $X$}

A geração X inclui os indivíduos nascidos entre 1965 e 1980 (Ordun, 2015). Esta foi uma geração que superou em número a geração precedente, os Baby Boomers (Mitchell, McLean \& Turner, 2005). A geração em estudo cresceu durante um período de grandes mudanças sociais (por exemplo, a legalização do aborto em alguns países, a liberalização do divórcio, um maior número de mulheres a entrar no mercado de trabalho, entre outras). Uma vez que as mães trabalhavam, as crianças passaram a frequentar as creches ou infantários, o que tornou a geração X mais independente e habituada a estar sozinha (Mitchell, McLean \& Turner, 2005). Ao nível ambiental, a geração X vivenciou alguns momentos marcantes, como a escassez das reservas de petróleo e consequentemente o aumento dos preços da energia, e a compra por parte de grandes empresas de áreas naturais, ameaçando os ecossistemas. Assistiu ao despertar das nações na questão de os recursos naturais serem limitados e poderem não estar disponíveis para as gerações futuras. Começaram a ser abordadas e divulgadas teorias sobre o aquecimento global, alertando para os riscos que o planeta vivia, bem como os impactos para a vida humana (Morton, 2003). A geração X é identificada como um complexo grupo de consumidores com pontos de vista, objetivos e desejos contraditórios. Esta geração cresceu com o hábito de ver televisão e os seus membros são envolvidos e responsáveis por grande parte das compras familiares. Dada a sua exposição à comunicação dos órgãos de comunicação tradicionais, têm conhecimento da manipulação das marcas e valorizam quando lhes são apresentados produtos de forma realista (Mitchell, McLean \& Turner, 2005).

Existem vários aspetos a ter em conta relativamente à comunicação e ao relacionamento com a geração X. A propaganda dirigida aos segmentos desta geração deve ter um conteúdo mais informativo do que persuasivo. Estes consumidores também gostam de websites que facultam as informações dos produtos que pretendem obter e apreciam a comunicação pessoal e o boca-a-boca (Morton, 2003). É mais difícil alcançar e convencer a geração $X$ do que a geração $Y$ através das redes sociais, pois esta é mais pragmática na avaliação dos comentários de outros usuários e meticulosa na escolha dos produtos (Dabija, Bejan \& Tipi, 2018).

Embora a geração $\mathrm{X}$ tenha crescido num momento de prosperidade econômica, revela ser sensível ao preço no momento de decisão de compra e procura o melhor valor para o seu dinheiro. Este é um importante fator que as marcas devem ter em conta na definição do preço, especialmente no mercado competitivo atual em que o cliente tem várias marcas à sua disposição. Além disso, como este é um consumidor com vontade de experimentar novos produtos, não é necessariamente fiel a uma marca. Os consumidores adultos comparativamente aos consumidores mais jovens, demonstram um padrão de consumo mais baseado na racionalidade e conhecimento, efetuam avaliações dos atributos 
do produto, processam a informação ambiental disponível e consideram o seu conhecimento ambiental (Lee, 2008). 0 altruísmo é também um dos fatores que influencia a decisão de compra na geração X. Para responder ao sentimento de altruísmo do consumidor, a política de comunicação das organizações deve considerar também as consequências positivas para as outras pessoas (Straughan \& Roberts, 1999).

\section{A geração $Y$}

A geração Y compreende os consumidores nascidos entre o período de 1981 a 2000 (Ordun, 2015). Cresceu testemunhando mudanças sociais como a diversidade cultural e étnica, uma consciencialização social intensificada e experimentaram já um mundo mais tecnológico e eletrônico, convivendo com computadores em casa e na escola. São jovens com um forte sentimento de autossuficiência, independência, autonomia e orientados para a aprendizagem (Williams \& Page, 2011; Parment, 2013). A aceitação de novas estruturas familiares e o endividamento através de créditos elevados, para cumprir os seus desejos e padrões de consumo, são familiares aos millennials (Parment, 2013).

Os consumidores da geração $Y$ vivem rodeados de informação, caracterizam-se por desempenhar várias tarefas em simultâneo, utilizam o seu telefone celular para tudo, particularmente para as redes sociais, para procurar um trabalho, informações sobre produtos, serviços ou até destinos de viagens, facto que potencia muitas oportunidades para o marketing online (Parment, 2013). Esta geração partilha as suas opiniões e recomendações com os seus amigos essencialmente através das redes sociais, como Facebook, Twitter e Instagram. Portanto, as marcas devem criar mais buzz marketing, conseguindo assim que os jovens falem da marca e a recomendem através de uma comunicação boca-a-boca positiva (Jang, Kim \& Bonn, 2011). É visível que para a geração Y a aceitação social tem um forte impacto nas suas escolhas ao nível da marca, do produto e até do risco que sentem quando escolhem um produto. Analisando o nível de envolvimento da geração Y com as marcas, no que respeita ao ambiente online, conclui-se que esta geração opta por escolher uma organização preferida com a qual cria a sua própria identidade, ignorando simplesmente as restantes organizações presentes no mercado (Rissanen \& Luoma-Aho, 2016).

Para os consumidores da geração Y é tão importante proteger o meio ambiente, através das suas opções de compra, como usufruir do produto. Portanto, o usufruto do produto é um importante fator que deve ser considerado nos produtos ecológicos. Considerar este fator irá tornar a adoção aos produtos ecológicos mais rápida e fortalecer a sustentabilidade no consumo (Gurtner \& Soyez, 2016). No seu estudo, Gurtner e Soyez (2016) identificaram um segmento de mercado de jovens consumidores ambientalmente conscientes, que desfrutam dos produtos que consomem, têm um papel ativo na criação de novas tendências e apresentam um estilo de vida baseado na inovação e ecologia. Por sua vez, para Joshi e Rahman (2016), os jovens consumidores evidenciam desejos de implementar na sociedade fortes mudanças ao nível de padrões de consumo responsáveis. Uma vez que estes jovens têm uma esperança média de vida alargada, pretendem assegurar que as mudanças introduzidas pela sua geração se mantenham por um longo período de tempo e passem para as gerações futuras. A geração Y revela também uma lacuna entre as suas atitudes pró-ambientais e o seu comportamento de compra ecológico. Portanto, a atitude pró-ambiental não é suficiente para desenvolver um comportamento de compra ecológico, sendo necessária uma compreensão mais profunda de outros possíveis fatores para este comportamento (Joshi \& Rahman, 2016), algo já identificado noutros estudos e segmentos de mercado, como no estudo de Pickett-Baker e Ozaki (2008).

As variáveis que influenciam o comportamento de compra dos consumidores da geração Y são (por ordem decrescente de importância): influência social, atitude em relação ao comportamento de compra ecológico, conhecimento ecológico percebido, participação no ato de reciclar, rótulos ecológicos e exposição às mensagens ecológicas através dos meios de comunicação social (Joshi \& Rahman, 2016). No que diz respeito aos rótulos dos produtos ecológicos, estes demonstram uma menor influência nos consumidores, o que se pode justificar pela falta de confiança nos mesmos e ceticismo em relação à produção, rotulagem e certificação dos produtos (Joshi \& Rahman, 2016). As 
campanhas de marketing verde devem focar-se em motivar os consumidores a abordar o tema de compra ecológica nos seus círculos sociais, uma vez que os millennials são influenciados pelos comportamentos de quem os rodeia. A organização de atividades ecológicas em grupo com os seus pais, amigos ou outros grupos, pode também influenciar o seu comportamento de compra ecológico (Joshi \& Rahman, 2016).

Os consumidores da geração Y devem ser persuadidos com mensagens que demonstrem que podem fazer a diferença e que têm capacidade de mudar o mundo. É ainda de considerar que esta geração está habituada a pensar também de forma racional e, por esse motivo, as comunicações de marketing ecológico devem ainda incluir apelos emocionais e racionais, utilizar meios de comunicação de massa e aumentar a frequência das mesmas (Joshi \& Rahman, 2016). A maioria dos jovens consumidores é de facto consumidora de produtos ecológicos. É também identificada neste segmento de mercado uma atitude positiva em relação às questões ecológicas e à aquisição de produtos ecológicos. Assim, é necessário que as organizações continuem a apostar em estratégias ecológicas e a encarar a sustentabilidade como uma política importante nos seus negócios, de forma a responder a estes segmentos de um modo eficaz. Por este motivo, é esperada uma expansão de mercado dos produtos ecológicos e que este mercado se torne também mais rentável (Kanchanapibul, Lacka, Wang \& Chan 2014).

\section{Estímulos de marketing}

Os benefícios emocionais associados às marcas podem ser utilizados como um encorajamento para os consumidores alterarem os seus padrões de consumo. Aliada à marca, a aplicação de estratégias de propaganda eficazes poderá causar mudanças nos consumidores que mostram disponibilidade para pagar um preço premium por produtos amigos do ambiente (Cherian \& Jacob, 2012). Juwaheer, Pudaruth e Noyaux (2012) salientam alguns pontos fulcrais no desenvolvimento de propaganda para o segmento ecológico, nomeadamente: a propaganda deve ser agradável, atrativa, convincente, diferenciadora e, ainda, incluir apelos emocionais relevantes para o contexto. A variável preço também influencia o comportamento do consumidor.

Quando um produto ecológico tem um preço considerado adequado, boa qualidade e não obriga a um sacrifício de conveniência por parte do consumidor, até os consumidores que não se identificam com comportamentos de compra ecológicos demonstram vontade de comprar esses produtos (Borin, Lindsey-Mullikin \& Krishnan, 2013). Contudo, tem-se verificado a introdução de produtos ecológicos no mercado a preços considerados justos. Esta é uma tendência que deve continuar devido à falta de consumidores disponíveis para pagar preços muito elevados por produtos amigos do ambiente. Portanto, uma empresa que queira introduzir produtos ecológicos no mercado não pode descurar a estratégia de preço (Borin, Lindsey-Mullikin \& Krishnan, 2013). Por sua vez, os rótulos ecológicos para além do principal objetivo de informar o cliente sobre as características do produto, auxiliam também no posicionamento, mostrando uma organização ambientalmente preocupada. Esta é uma forma eficaz de comunicar ao cliente os benefícios específicos do produto e as suas características ambientalmente seguras, recorrendo a mensagens ambientais (D’Souza, Taghian \& Peretiakos, 2006).

Apesar de a informação estar disponível nos rótulos e o consumidor a ler, parece não a considerar exata ou fácil de perceber, podendo não ser a mais clara e criar confusão. Desta forma, D'Souza et al. (2006) consideram que os rótulos das embalagens ecológicas não comunicam a informação do produto de forma eficaz. Sugerem algumas melhorias para facilitar a comunicação, designadamente evitar o uso de termos técnicos, nomes em código e identificar gráfica e distintamente os aspetos amigos do ambiente que o produto cumpre, de forma a providenciar informação compreensível para o consumidor (D'Souza et al., 2006; Juwaheer, Pudaruth \& Noyaux, 2012). 0 conhecimento dos consumidores pode ser estimulado através dos rótulos ecológicos, tornando estes produtos facilmente reconhecíveis e, ao mesmo tempo, criando credibilidade, sendo que, para tal, devem estar presentes certificações de entidades externas (Dangelico \& Pujari, 2010; Kong, Harun, Sulong \& Lily, 2014). 
A propaganda é uma oportunidade para aumentar o conhecimento e a familiaridade dos consumidores com os produtos ecológicos. Porém, com alguma cautela, pois um dos fatores pelos quais a propaganda falha em originar comportamentos de compra ecológicos é a sua falta de credibilidade entre os consumidores (Delafrooz, Taleghani \& Nouri, 2014). 0 estudo de Delafrooz, Taleghani e Nouri (2014) indica que a propaganda é um dos fatores com mais influência na previsão do comportamento de compra ecológico, seguida dos rótulos ecológicos, e o fator com menos impacto são as marcas ecológicas. Assim, os autores são da opinião que devem ser feitos esforços para continuar a informar o público sobre questões ambientais e aumentar o conhecimento sobre produtos que são menos prejudiciais ao meio ambiente. Uma outra forma de aumentar a popularidade deste tipo de produtos no mercado, para além do recurso à propaganda, será baixando os preços e aumentando a variedade disponível (Delafrooz, Taleghani \& Nouri, 2014). Todavia, os resultados do estudo de Rahbar e Wahid (2011) indicam que a propaganda é identificada como uma ferramenta de marketing verde útil na criação de conhecimento sobre o tema, mas sem influência no comportamento de compra de produtos ecológicos.

\section{HIPÓTESES DE INVESTIGAÇÃO}

O referencial teórico apresentado aponta para algumas diferenças entre os consumidores das gerações $\mathrm{X}$ e $\mathrm{Y}$, sendo necessário aprofundar se, de facto, as gerações mencionadas demonstram atitudes e comportamentos ecológicos diferenciados.

De acordo com Roberts (1996), a geração X demonstra possuir uma consciência ambiental aquando da compra, sendo o altruísmo um dos fatores que influencia a sua decisão (Straughan \& Robert, 1999). Os consumidores mais velhos, comparativamente aos consumidores mais jovens, demonstram um padrão de consumo mais baseado na racionalidade e conhecimento, efetuam avaliações dos atributos do produto e processam a informação ambiental disponível considerando o seu conhecimento ambiental (Lee, 2008). Com base nestes argumentos prevê-se uma maior predisposição para o comportamento ecológico por parte da geração X. Deste modo, formula-se a seguinte hipótese: H1: Os consumidores da geração X estão mais predispostos a adquirir produtos com um reduzido impacto ambiental, do que os consumidores da geração Y.

Normalmente a geração $\mathrm{X}$ procura e considera cuidadosamente a informação sobre os produtos, marcas e serviços, é preocupada, minuciosa e atenta na procura de informação sobre as características do produto (Dabija, Bejan \& Tipi, 2018). Como já referido anteriormente, os consumidores mais maduros, comparativamente aos consumidores mais jovens, processam a informação ambiental disponível considerando o seu conhecimento ambiental, estando por isso aptos a identificar os produtos mais amigos do ambiente (Lee, 2008). Desta forma, formula-se a segunda hipótese deste estudo: H2: Os consumidores da geração $\mathrm{X}$ conseguem identificar os produtos ecológicos mais facilmente do que os da geração Y.

Autores como Jang, Kim e Bonn (2011) defendem que ambas as gerações analisam, pesquisam e consideram cuidadosamente as informações que obtém sobre os produtos antes de os comprar. Contudo, os consumidores da geração Y revelam atribuir mais importância às informações específicas dos produtos disponibilizadas através dos rótulos como, por exemplo, a origem e o seu valor nutritivo (Jang, Kim \& Bonn, 2011). Para além destes fatores, sabemos que a geração Y é a geração que melhor encara e lida com excesso de informação, enquanto para outras gerações, como a X, este gerenciamento de informação pode ser desgastante (Parment, 2013). Assim, formula-se a seguinte hipótese: H3: Para a geração Y os rótulos comunicam de forma mais eficaz as características ecológicas dos produtos, do que para a geração $X$.

A propaganda dirigida à geração $\mathrm{X}$ deve ter um conteúdo mais informativo do que persuasivo e ser um marco de irreverência (Mitchell, McLean \& Turner, 2005). A propaganda dirigida à geração $Y$ deve cumprir alguns pontos importantes: apelar à crença de que esta geração irá tornar o futuro melhor, comunicar exemplos da vida real, valorizar a verdade e a realidade e incluir a diversidade de raças e etnias. Os anúncios devem ser únicos e distinguíveis, conter informação sobre o produto, honestidade e humor (Williams \& Page, 2011). Assim, a geração Y é a que parece relacionar-se e responder melhor à propaganda com apelos emocionais. Por este motivo, propõe-se testar a seguinte 
hipótese: H4: As campanhas de comunicação com forte apelo emocional terão um maior impacto na intenção de compra de produtos ecológicos na geração $\mathrm{Y}$ do que na geração $\mathrm{X}$.

Tanto a geração X como a Y demonstram sensibilidade ao fator preço. A geração X revela uma grande sensibilidade ao preço, procura o melhor valor pelo seu dinheiro, sendo um dos segmentos de mercado mais conscientes relativamente ao preço dos produtos (Williams \& Page, 2011). Os millennials esperam preços competitivos e podem até negociar preços com base na propaganda que viram na concorrência ou até nas páginas da internet. Ainda assim, revelam uma elevada propensão para a compra de produtos de prestígio (Williams \& Page, 2011), para além de que é identificado na geração Y um elevado poder de compra (Jang, Kim \& Bonn, 2011; Ordun, 2015). A geração Y, quando associa prestígio e melhores características ao produto, está disponível a pagar mais pelo mesmo. Desta forma, identifica-se neste grupo de consumidores disponibilidade para pagar mais por determinados produtos. Portanto, ir-se-á testar a possibilidade de o preço não ser um impedimento para a compra de produtos ecológicos para a geração $\mathrm{Y}$, formulando-se a última hipótese de estudo: H5: Para os consumidores da geração Y o fator preço não é um impedimento à compra de produtos ecológicos, ao contrário da geração X.

\section{METODOLOGIA}

A pesquisa em causa é do tipo descritivo, dado que pretende estudar um fenômeno de mercado e a influência dos estímulos de marketing ecológico no comportamento de compra do consumidor português. Este estudo caracteriza-se também por ser inferencial, dado que utiliza os dados recolhidos na amostra disponível para obter conclusões sobre a correspondente população, formada por todos os consumidores portugueses pertencentes às gerações $\mathrm{X}$ e $\mathrm{Y}$.

Para analisar o fenômeno em estudo foi utilizada uma amostra por conveniência (Hill \& Hill, 2016), mas foi cumprido o critério de pertença a uma das duas gerações em estudo. A amostra considerada neste estudo conta com um total de 309 inquiridos, dos quais 145 pertencem à geração $\mathrm{X}$ e 164 à geração Y. Contabilizaram-se ainda 48 inquiridos que não pertenciam a nenhuma destas gerações e, por esse motivo, não foram considerados. No total da amostra, $65.69 \%$ dos inquiridos pertence ao sexo feminino e $34.31 \%$ ao masculino. Constata-se que $39.78 \%$ dos inquiridos é casada, $52.55 \%$ solteira e $7.66 \%$ responderam "outro". Verifica-se que a maioria dos inquiridos tem como habilitações literárias uma licenciatura, sendo $42.70 \%$ da amostra, seguido do $12^{\circ}$ ano, com $32.85 \%$. 0 mestrado é a terceira opção com maior taxa de resposta, a saber, $14.96 \%$.

0 questionário foi o método de recolha de dados deste estudo, tendo sido previamente testado num grupo de indivíduos cujas sugestões foram incorporadas na versão final do mesmo. A partilha do questionário foi através das redes sociais, tendo alcançado os utilizadores destas redes, identificados por Malhotra (2011) como um dos grupos de amostragem de conveniência. O questionário foi composto por 59 questões e por várias secções, iniciando-se com uma questão filtro para impedir que inquiridos que não pertencessem às gerações em estudo respondessem. Posteriormente, foi apresentada uma secção cujo objetivo medir os hábitos de consumo ecológico e a periodicidade de compra de produtos ecológicos, recorrendo a uma escala de Likert de 5 pontos.

A secção seguinte visava perceber se os consumidores verificavam as características ecológicas dos produtos e de que forma o faziam. Recorreu-se, para este efeito, a questões dicotômicas, a um conjunto de perguntas com a escala de Likert e uma resposta aberta. De seguida, foram colocadas questões relacionando as variáveis de marketing mix com o contexto ecológico, especificamente aspetos relacionados com o rótulo, a distribuição, a embalagem, o preço e a propaganda, apenas através da escala de Likert.

Foram vários os trabalhos que serviram de base ao desenho deste questionário, e que auxiliaram na construção dos construtos que serviram de suporte à formação das hipóteses, como sejam, as escalas apresentadas em Straughan e Robert (1999), Pickett-Baker e Ozaki (2008), Delafrooz et al. (2014) e Joshi e Rahman (2016). Na última seç̧ão, fez-se o levantamento dos dados sociodemográficos dos inquiridos (gênero, estado civil, nível de escolaridade, rendimento e se tem filhos) através de perguntas dicotômicas e de escolha múltipla. A partilha do questionário final foi feita através das redes sociais, mas antes foi realizado um pré-teste com uma amostra com doze 
consumidores pertencentes a ambas as gerações, para perceber se o instrumento de recolha dos dados era claro e intuitivo.

Por fim, refira-se que a análise de dados foi realizada com recurso ao software IBM SPSS Statistics 25. É de notar que as variáveis ordinais deste estudo podem ter tratamento quantitativo, porque, conforme será visto mais adiante, têm mais de três categorias (Pestana \& Gageiro, 2014). Assim, é possível calcular médias destas variáveis nas gerações X e Y e compará-las através da utilização do teste t para amostras independentes (Pestana \& Gageiro, 2014). Este teste é usado sempre que se pretende comparar duas médias em dois grupos diferentes de sujeitos e se desconhecem as respetivas variâncias populacionais, como é o caso aqui. A estatística de teste t pode ter duas expressões diferentes, consoante as variâncias populacionais possam ou não ser assumidas como iguais, conclusão que se obtém do teste de Levene (Pestana \& Gageiro, 2014).

0 teste $\mathrm{t}$ para amostras independentes pode ser aplicado se a distribuição das médias amostrais for normal ou aproximadamente normal. Para tal, as variáveis cujas médias se pretende comparar deverão ter distribuição normal ou, independentemente da distribuição dessas variáveis, os tamanhos das amostras deverão ser superiores a 30, caso em que se aplica o teorema do limite central (Pestana \& Gageiro, 2014). Neste estudo, verificou-se que a distribuição das variáveis não é normal, uma que o valor de prova do teste de Kolmogorov-Smirnov com a correção de Lilliefors (Pestana \& Gageiro, 2014) foi sempre inferior a 0,001. No entanto, como a amostra da geração X é formada por 145 indivíduos e a da geração Y por 164 indivíduos, ambas têm dimensão superior a 30 e o teste $\mathrm{t}$ pode ser aplicado. 0 nível de significância fixado foi de 5\%. A Tabela 1 no final da próxima secção agrega os resultados apresentados ao longo da mesma.

\section{ANÁLISE E DISCUSSÃO DOS RESULTADOS Teste da hipótese 1}

H1: Os consumidores da geração $\mathrm{X}$ estão mais predispostos a adquirir produtos com um reduzido impacto ambiental, do que os consumidores da geração Y.

No teste desta hipótese, os dados considerados foram os recolhidos no âmbito da questão "Até que ponto as questões ambientais são fatores que o/a impulsionam a comprar certos produtos?" (Questão 1, Q1), tendo sido as respostas dos inquiridos sujeitas a uma escala de 1 (Nada) a 5 (Bastante). A pergunta permitiu avaliar diretamente a importância que os consumidores dão às questões ambientais na compra de produtos e esta importância revela-nos a predisposição que o consumidor tem para comprar produtos ecológicos. Em termos amostrais, verificou-se o seguinte em relação às respostas nos dois grupos (média +/- desvio-padrão): 3,58 +/- 1,025 para a geração X e 3,48 +/- 0,936 para a Y. Assim, comparando os valores médios das amostras, conclui-se que a predisposição para adquirir produtos ecológicos tende a ser maior nos inquiridos da geração X.

Testar a hipótese $\mathrm{H} 1$ consiste em testar se a média populacional na geração X é maior que a média populacional na geração Y (teste unilateral direito). Assumindo que as variâncias nas duas populações são iguais (o valor de prova do teste de Levene é valor-p=0,201>0,05), realizou-se um teste $t$ de amostras independentes, tendo-se concluído que valor- $\mathrm{p}=0,1765$. Deste modo, como o valorp $>0,05$, não se aceita H1, isto é, não há evidências estatísticas suficientes de que os consumidores da geração X estejam mais predispostos a adquirir produtos com um reduzido impacto ambiental, comparativamente com os consumidores da geração Y. O estudo de Roberts (1996) concluiu que a geração X tinha uma maior consciência ambiental.

Lee (2008) verificou que os consumidores mais velhos demonstram um padrão de consumo mais baseado na racionalidade e conhecimento, efetuam avaliações dos atributos do produto e processam a informação ambiental disponível, considerando o seu conhecimento neste domínio. Por outro lado, são também vários os estudos que evidenciam os comportamentos ecológicos da geração Y, como o de Kanchanapibul et al. (2014), que verificaram a existência de uma atitude positiva em relação a questões ecológicas e aquisição de produtos ecológicos por parte desta geração. Joshi e Rahman (2016), para além de identificarem uma atitude positiva em relação a comportamentos de 
compra ecológicos, identificaram uma sensibilidade em relação aos efeitos dos seus padrões de consumo e um pensamento racional acerca dos benefícios das suas compras. Existem, portanto, estudos que sustentam a predisposição para a compra ambiental de ambas as gerações. No caso do presente trabalho, concluiu-se que os consumidores das duas gerações estão igualmente predispostos a adquirir produtos com um reduzido impacto ambiental. Verificou-se, ainda, um consumo moderado de produtos ecológicos em ambas as gerações, dado que, em ambos os grupos, a maioria respondeu que compra produtos ecológicos "às vezes". Além disso, constatou-se que, quanto maior a convicção ambiental dos inquiridos, maior tendia a ser a compra de produtos ecológicos (correlação Tau-b de Kendall $=0,465$, valor $-\mathrm{p}=0,000$ ).

\section{Teste da hipótese 2}

H2: Os consumidores da geração X conseguem identificar os produtos ecológicos mais facilmente do que os da geração $\mathrm{Y}$.

No teste desta segunda hipótese, os dados considerados foram os recolhidos no âmbito da questão "Até que ponto consegue identificar os produtos que são menos prejudiciais para o meio ambiente?" (Questão 2, Q2), tendo sido as respostas dos inquiridos baseadas numa escala de 1 (Nada) a 5 (Bastante). Optou-se por esta pergunta uma vez que questiona diretamente os indivíduos sobre a sua capacidade de identificação dos produtos ecológicos. Em termos amostrais, verificou-se o seguinte em relação às respostas nos dois grupos: $3,52+/-0,800$ para a geração $\mathrm{X}$ e 3,41 +/- 0,734 para a Y. Assim, comparando os valores médios das amostras, conclui-se que a capacidade para identificar produtos ecológicos tende a ser maior nos inquiridos da geração X.

Tal como no caso da hipótese H1, testar a hipótese H2 consiste em testar se a média populacional na geração X é maior que a média populacional na geração $\mathrm{Y}$ (teste unilateral direito). Assumindo que as variâncias nas duas populações são iguais (no teste de Levene obteve-se valor$\mathrm{p}=0,431>0,05)$, realizou-se um teste $\mathrm{t}$ de amostras independentes, tendo-se concluído que valor$\mathrm{p}=0,1055$. Deste modo, como valor-p>0,05, não se aceita $\mathrm{H} 2$, isto é, não há evidências estatísticas suficientes de que os consumidores da geração $X$ consigam identificar os produtos ecológicos mais facilmente do que os da geração Y. Quanto maior for a compreensão dos rótulos e a identificação dos produtos ecológicos pelo rótulo, mais fácil será a identificação de produtos ecológicos quando o consumidor faz as suas compras (Rahbar \& Wahid, 2011). Como referido no estudo de Juwaheer, Pudaruth e Noyaux (2012), existe uma relação positiva entre a disponibilidade de compra de produtos ecológicos e a facilidade de leitura dos rótulos, sendo é importante a identificação dos mesmos. Assim, a identificação dos produtos ecológicos está diretamente relacionada com a fácil compreensão dos rótulos destes produtos (Rahbar \& Wahid, 2011). Neste trabalho, concluiu-se que a facilidade em identificar produtos ecológicos é similar em ambas as gerações.

\section{Teste da Hipótese 3}

H3: Para a geração Y os rótulos comunicam de forma mais eficaz as características ecológicas dos produtos, do que para a geração $X$.

No teste desta terceira hipótese, os dados considerados foram os recolhidos no âmbito da afirmação "Pelo rótulo percebo que é um produto ecológico." (Afirmação 1, A1), tendo sido as opiniões dos inquiridos medidas numa escala de 1 (Discordo completamente) a 5 (Concordo completamente). A opinião dos indivíduos permite saber se o rótulo possibilita ao consumidor identificar se o produto é ecológico ou não. Em termos amostrais, verificou-se o seguinte em relação às respostas nos dois grupos: $3,44+/-0,815$ para a geração $\mathrm{X}$ e $3,23+/-0,982$ para a $\mathrm{Y}$. Assim, comparando os valores médios das amostras, conclui-se que os inquiridos da geração $Y$ tendem a concordar menos com a afirmação de que o rótulo permite perceber que o produto é ecológico e isto sugere que a hipótese enunciada não se verifica. Enquanto os testes das duas hipóteses anteriores eram unilaterais direitos, 
o teste da hipótese H3 é unilateral esquerdo, dado que se pretende testar se a média populacional na geração Y é maior que a média populacional na geração X.

Assumindo que as variâncias nas duas populações diferem (no teste de Levene obteve-se valor-p=0,016 $<0,05)$, realizou-se um teste $t$ de amostras independentes, tendo-se concluído que valor$\mathrm{p}=0,9795$. Deste modo, dado o valor-p>0,05, não se aceita $H 3$, isto é, não há evidências estatísticas suficientes de que os rótulos comuniquem de forma mais eficaz as características ecológicas dos produtos para a geração Y. Ao nível da influência e percepção dos rótulos, existem alguns estudos. Por um lado, analisou-se o estudo de Kong et al. (2014), onde se verificou que a informação disponível nos rótulos era considerada de fácil compreensão e precisa. Por outro lado, o estudo de D'Souza et al. (2006), chegou à conclusão de que os consumidores não consideram os rótulos exatos ou fáceis de entender, o que na opinião destes autores indica que os rótulos ecológicos não comunicam a informação do produto de forma eficaz. No caso do presente trabalho, concluiu-se que a capacidade de se perceber pelo rótulo que um produto é ecológico é a mesma nas duas gerações.

\section{Teste da Hipótese 4}

H4: As campanhas de comunicação com forte apelo emocional terão um maior impacto na intenção de compra de produtos ecológicos na geração $\mathrm{Y}$ do que na geração $\mathrm{X}$.

No teste desta quarta hipótese, os dados considerados foram os recolhidos no âmbito da afirmação "Um anúncio publicitário com um apelo emocional forte motiva-me a comprar esse produto." (Afirmação 2, A2), tendo sido as opiniões dos inquiridos analisadas com base numa escala de 1 (Discordo completamente) a 5 (Concordo completamente). Em termos amostrais, verificou-se o seguinte em relação às respostas nos dois grupos: 3,27 +/- 1,101 para a geração $X$ e 3,32 +/- 1,074 para a Y. Assim, comparando os valores médios das amostras, conclui-se que os inquiridos da geração Y tendem a concordar ligeiramente mais com a afirmação de que um anúncio publicitário com um apelo emocional forte motiva a comprar esse produto. Tal como no caso da hipótese H3, testar a hipótese $\mathrm{H} 4$ consiste em testar se a média populacional na geração $\mathrm{Y}$ é maior que a média populacional na geração X (teste unilateral esquerdo).

Assumindo que as variâncias nas duas populações são iguais (no teste de Levene obteve-se valor-p=0,725 $>0,05$ ), realizou-se um teste $t$ de amostras independentes, tendo-se concluído que valor$\mathrm{p}=0,331$. Deste modo, como valor-p $>0,05$, não se aceita $\mathrm{H} 4$, isto é, não há evidências estatísticas suficientes de que as campanhas de comunicação com forte apelo emocional tenham um maior impacto na intenção de compra de produtos ecológicos na geração Y. Na sequência do teste realizado, analisaram-se os dados recolhidos no âmbito de outra afirmação, relacionada com a frequência de consumo, designadamente, "Este apelo emocional faz com que compre o produto mais do que uma vez." (Afirmação 3, A3), em que as opções de resposta estavam definidas numa escala de 1 (Discordo completamente) a 5 (Concordo completamente).

Considerou-se também esta afirmação, pois se entendeu ser relevante perceber se há uma diferença entre as duas gerações no que respeita à repetição do comportamento de compra provocado pela comunicação com apelos emocionais. Nas amostras, verificou-se que há apenas uma ligeira diferença $(0,01)$ entre os valores médios das respostas nos dois grupos, ou seja, entre a opinião geral dos inquiridos das duas gerações no que respeita à frequência de compra. Para aferir se a diferença é estatisticamente significativa, realizou-se um teste $t$ de amostras independentes e o valor-p do teste bilateral é 0,921. Assim, como valor-p>0,05, não há evidências estatísticas suficientes de que haja uma diferença entre as duas gerações no que respeita à repetição do comportamento de compra provocado pela comunicação com apelos emocionais. Segundo Juwaheer, Pudaruth e Noyaux (2012), um dos pontos fulcrais para as comunicações de marketing ecológico é incluir apelos emocionais relevantes para o contexto.

No caso da geração $Y$, esta revela ser muito receptiva a comunicações que incluem apelos emocionais (Joshi \& Rahman, 2016). Assim, os apelos emocionais devem ser incluídos nas comunicações dirigidas à geração $\mathrm{Y}$; porém, a geração $\mathrm{X}$ também demonstra responder positivamente 
a estes apelos. As comunicações de marketing ecológico serão mais bem-sucedidas se incluírem ambos os apelos emocionais e racionais, pois estes segmentos de consumidores estão recetivos a tais mensagens. Conjugando estes apelos através da comunicação das marcas com a predisposição de compra de produtos ecológicos identificada anteriormente, estarão reunidas condições para reduzir a lacuna entre atitudes e convicções ecológicas dos consumidores portugueses e aumentar o consumo de produtos ecológicos. No presente estudo, chegou-se à conclusão de que as campanhas de comunicação com forte apelo emocional têm um impacto similar na intenção de compra de produtos ecológicos para as gerações X e Y.

\section{Teste da Hipótese 5}

H5: Para os consumidores da geração $\mathrm{Y}$ o fator preço não é um impedimento à compra de produtos ecológicos, ao contrário da geração X.

Conforme é sabido, os produtos ecológicos são, normalmente, mais caros do que os produtos convencionais, o que é considerado um entrave à sua aquisição. Assim sendo, e dado que é um tópico essencial no consumo de qualquer categoria de produto, mas especialmente nesta categoria de produtos, pretende-se saber a disponibilidade de cada geração para pagar mais por um produto ecológico. Desta forma, no teste desta hipótese H5, consideraram-se os dados recolhidos no âmbito da afirmação "Estou disponível para pagar mais por um produto com um impacto ambiental menor." (Afirmação 4, A4), tendo sido as opiniões dos inquiridos analisadas numa escala de 1 (Discordo completamente) a 5 (Concordo completamente). Comparando os valores médios das amostras, conclui-se que os inquiridos da geração Y mostram-se tendencialmente mais disponíveis para pagar por um produto com um impacto ambiental menor (respostas: 3,05 +/- 0,988 para a geração X e 3,29 +/- 1,039 para a Y). Tal como nos testes das duas hipóteses anteriores, foi aplicado um teste unilateral esquerdo. Assumindo que as variâncias nas duas populações são iguais (no teste de Levene obteve-se valor- $\mathrm{p}=0,437>0,05$ ), realizou-se um teste $\mathrm{t}$ de amostras independentes, tendo-se concluído que valor$\mathrm{p}=0,018$. Deste modo, como valor-p $<0,05$, aceita-se H5, isto é, há evidências estatísticas suficientes de que, para a geração $\mathrm{Y}$, o preço não é um impedimento à compra de produtos ecológicos, ao contrário do que acontece com a geração X. Verificou-se adicionalmente que, quanto maior é a importância que o inquirido atribui às características ecológicas de um produto, enquanto critério de compra, maior tende a ser a sua disponibilidade para pagar mais por um produto ecológico (correlação Tau-b de Kendall $=0,426$, valor-p=0,01). Logo, quanto maior forem as preocupações ambientais, maior será a disponibilidade para comprar produtos ecológicos mais caros, conclusão que vai ao encontro do estudo de Akehurst, Afonso \& Gonçalves (2012). Pela revisão de literatura, concluiu-se que os millennials esperam preços competitivos, mas revelam uma elevada probabilidade de comprar produtos caros (Williams \& Page, 2011). Relativamente à geração X, é reconhecida por vários autores (Williams \& Page, 2011) uma elevada sensibilidade ao preço e, como tal, esta geração procura sempre o melhor valor pelo seu dinheiro. Neste trabalho, concluiu-se que a tendência para pagar mais por um produto com um impacto ambiental menor é maior na geração Y.

Resumindo, a Tabela 1 a seguir apresenta as principais estatísticas e as decisões relacionadas com os testes das hipóteses consideradas. Com efeito, e resumindo os resultados encontrados, podemos verificar que os consumidores das duas gerações ( $\mathrm{X}$ e $\mathrm{Y}$ ) estão igualmente predispostos a adquirir produtos com um reduzido impacto ambiental, ainda que haja estudos que sustentam que os consumidores X são mais ecológicos (Lee, 2008; Roberts, 1996) e outros que atestam o mesmo resultado em relação aos consumidores Y (Kanchanapibul et al., 2014). 0 mesmo ocorre no caso da facilidade em identificar produtos ecológicos e na capacidade de se perceber pelo rótulo que um produto é ecológico, que também tendem a ser similares em ambas as gerações, apesar de haver estudos que indicam que os consumidores da geração $\mathrm{Y}$ revelam atribuir mais importância às informações específicas dos produtos disponibilizadas através dos rótulos como, por exemplo, a origem e o seu valor nutritivo (Jang, Kim \& Bonn, 2011). Do mesmo modo, como já foi referido 
anteriormente, a geração Y é a geração que melhor encara e lida com excesso de informação, sendo que para a geração X, este gerenciamento de informação pode ser desgastante (Parment, 2013).

\section{Tabela 1}

Principais estatísticas e decisões (Significância de 5\%) relacionadas com os testes das hipóteses consideradas

\begin{tabular}{|c|c|c|c|c|c|c|c|c|c|c|}
\hline \multirow{2}{*}{ Hipótese } & \multirow{2}{*}{ Questão } & \multirow{2}{*}{ Geração } & \multicolumn{2}{|c|}{$\begin{array}{c}\text { Estatísticas de } \\
\text { grupo }\end{array}$} & \multicolumn{2}{|c|}{$\begin{array}{c}\text { Teste de } \\
\text { Levene para a } \\
\text { igualdade de } \\
\text { variâncias }\end{array}$} & \multicolumn{3}{|c|}{$\begin{array}{c}\text { Teste T para } \\
\text { comparação das } \\
\text { médias }\end{array}$} & \multirow{2}{*}{$\begin{array}{c}\text { Decisão } \\
\text { sobre a } \\
\text { hipótese }\end{array}$} \\
\hline & & & Média & $\begin{array}{l}\text { Desvio- } \\
\text { padrão }\end{array}$ & $\mathbf{F}$ & $\mathbf{p}$ & $\mathbf{T}$ & $\mathbf{G L}^{*}$ & $\mathbf{p}$ & \\
\hline H1 & Q1 & $\begin{array}{l}X \\
Y\end{array}$ & $\begin{array}{l}3,58 \\
3,48\end{array}$ & $\begin{array}{l}1,025 \\
0,936\end{array}$ & 1,640 & 0,201 & 0,929 & 307 & 0,176 & Não aceitar \\
\hline $\mathrm{H} 2$ & Q2 & $\begin{array}{l}\mathrm{X} \\
\mathrm{Y}\end{array}$ & $\begin{array}{l}3,52 \\
3,41\end{array}$ & $\begin{array}{l}0,800 \\
0,734\end{array}$ & 0,622 & 0,431 & 1,255 & 307 & 0,105 & Não aceitar \\
\hline H3 & $\mathrm{A} 1$ & $\begin{array}{l}X \\
Y\end{array}$ & $\begin{array}{l}3,44 \\
3,23\end{array}$ & $\begin{array}{l}0,815 \\
0,982\end{array}$ & 5,889 & 0,016 & 2,049 & 305 & 0,979 & Não aceitar \\
\hline \multirow{2}{*}{$\mathrm{H} 4$} & A2 & $\begin{array}{l}X \\
Y\end{array}$ & $\begin{array}{l}3,27 \\
3,32\end{array}$ & $\begin{array}{l}1,101 \\
1,074\end{array}$ & 0,124 & 0,725 & $-0,438$ & 307 & 0,331 & \multirow{2}{*}{ Não aceitar } \\
\hline & A3 & $\begin{array}{l}X \\
Y\end{array}$ & $\begin{array}{l}2,94 \\
2,93\end{array}$ & $\begin{array}{l}1,026 \\
1,069\end{array}$ & 0,549 & 0,459 & 0,100 & 307 & 0,921 & \\
\hline H5 & A4 & $\begin{array}{l}X \\
Y\end{array}$ & $\begin{array}{l}3,05 \\
3,29\end{array}$ & $\begin{array}{l}0,988 \\
1,039\end{array}$ & 0,605 & 0,437 & $-2,111$ & 307 & 0,018 & Aceitar \\
\hline
\end{tabular}

Nota: *GL - Graus de Liberdade

Adicionalmente, este estudo concluiu que as campanhas de comunicação com forte apelo emocional têm um impacto similar na intenção de compra de produtos ecológicos para ambas as gerações. No entanto, este resultado poderia ter sido diferente, dependendo do tipo de campanha indicado, já que há estudos que evidenciam que a comunicação social é já bem conhecida pela geração $\mathrm{X}$, por isso é tratada com irreverência e ironia e, no caso do lançamento de novas campanhas, não é um método eficaz de alcançar este público. Logo, a melhor forma de comunicação para a geração X é a comunicação pessoal e o boca-a-boca (Morton, 2003). Paralelamente, Joshi e Rahman (2016) argumentam que os consumidores da geração $\mathrm{Y}$ devem ser persuadidos com mensagens que demonstrem que podem fazer a diferença e que têm capacidade de mudar o Mundo. Finalmente, a única hipótese que realmente se confirmou neste estudo é a que evidencia que para os consumidores da geração Y o fator preço não é um impedimento à compra de produtos ecológicos, ao contrário da 
geração X, o que vai ao encontro dos estudos que indicam que a geração X é reconhecida por ter uma elevada sensibilidade ao preço (Williams \& Page, 2011).

\section{CONCLUSÃO}

O objetivo desta pesquisa foi estudar o efeito de algumas variáveis (propaganda, informação veiculada nos rótulos e preço) no comportamento de compra do consumidor. No que respeita aos rótulos dos produtos ecológicos, os resultados encontrados em ambas as gerações são semelhantes, mas como a identificação dos produtos ecológicos está diretamente relacionada com a fácil compreensão dos rótulos destes produtos (Rahbar \& Wahid, 2011), sugere-se que as empresas tenham uma atenção particular nesta matéria. Outro aspeto essencial é o preço dos produtos ecológicos, pois embora a geração Y esteja disponível a pagar mais por um produto ecológico, é do conhecimento geral que o preço é sempre um aspeto sensível para os consumidores (Kianpour, Jusoh \& Asghari, 2012). Logo, as marcas também devem ter este fator em consideração, não devendo concluir que um consumidor ecológico está sempre disposto a pagar um preço maior por um produto ecológico.

Por fim, no que respeita à propaganda, é preciso considerar que, embora as gerações $\mathrm{X}$ e $\mathrm{Y}$ tenham respondido de forma semelhante, segundo a literatura, esta resposta pode variar conforme os estímulos e os meios de comunicação envolvidos. Por exemplo, a geração X privilegia mais a comunicação pessoal (Morton, 2003) e informativa (Mitchell, McLean \& Turner, 2005) e a Y a comunicação digital (Parment, 2013) e em massa, que inclua apelos emocionais (Joshi \& Rahman, 2016). Logo, as empresas precisam de compreender a importância de traçar uma estratégia de Marketing (Toaldo \& Luce, 2006), sobretudo no que diz respeito à sua comunicação, a qual deve integrar vários modelos estratégicos de Marketing, como o de Noble e Mokwa (1999). De resto, espera-se que as marcas utilizem cada vez mais ferramentas que lhes permitam segmentar o mercado devidamente, para conseguirem comunicar de um modo mais personalizado com consumidores que têm algo em comum, através, por exemplo, das redes sociais, como o Facebook (Ferreira \& Zambaldi, 2019).

Como limitação do estudo, destaca-se a não generalização dos seus resultados, pois a amostra utilizada foi do tipo não probabilística por conveniência. Porém, é inovador, dado que não foram encontradas evidências de que um estudo deste tipo já tenha sido aplicado em contexto português, servindo de base às empresas que pretendem atuar no mercado dos produtos ecológicos. Paralelamente, este estudo indica que a variável que verdadeiramente faz diferença no consumo de produtos, nomeadamente ecológicos, é o preço, o que pode ajudar as marcas a trabalhar este P do mix de marketing de forma mais produtiva e direcionada.

Para estudos futuros sugere-se investigar se a baixa percepção do benefício dos produtos ecológicos por parte dos consumidores é uma razão que justifica o baixo envolvimento na compra ecológica. Outra sugestão é o estudo de variáveis que possam influenciar positivamente os consumidores que ainda não compram produtos ecológicos, a adotar esta forma de comportamento.

\section{Agradecimentos}

Este trabalho foi parcialmente financiado pela Fundação para a Ciência e Tecnologia (FCT), através do Centro de Investigação e Desenvolvimento em Matemática e Aplicações (CIDMA), no âmbito dos projetos UIDB/04106/2020 e UIDP/04106/2020.

\section{Referências}

Akehurst, G., Afonso, C., \& Gonçalves, H. M. (2012). Re-examining green purchase behaviour and the green consumer profile: new evidences. Management Decision, 50(5), 972-988. https://doi.org/10.1108/00251741211227726

Borin, N., Lindsey-Mullikin, J., \& Krishnan, R. (2013). An analysis of consumer reactions to green strategies. Journal of Product \& Brand Management, 22(2), 118-128. https://doi.org/10.1108/10610421311320997] 
Cherian, J., \& Jacob, J. (2012). Green Marketing: A Study of Consumers' Attitude towards Environment Friendly Products. Asian Social Science, 8(12), 117-126. https://doi.org/10.5539/ass.v8n12p117

Ciribele, J. P., \& Caneschi, B. da S. (2011). Marketing verde: um diferencial competitivo ou uma questão essencial para as empresas sobreviverem no século XXI. Revista Gestão Empresarial, 1(1), 114125

Côrtes, P. L. \& Moretti, S. L. A. (2013). Consumo verde: um estudo transcultural sobre crenças, preocupações e atitudes ambientais. REMark -Revista Brasileira de Marketing, 12(3), 45-76. https://doi.org/10.5585/remark.v12i3.2592

Dabija, D.-C., Bejan, B. M., \& Tipi, N. (2018). Generation X versus Millennials communication behavior on social media when purchasing food versus tourist services. Marketing and Trade, 21(1), 191205. https://doi.org/10.15240/tul/001/2018-1-013

Dangelico, R. M., \& Pujari, D. (2010). Mainstreaming Green Product Innovation: Why and How Companies Integrate Environmental Sustainability. Journal of Business Ethics, 95(3), 471-486. https://doi.org/10.1007/s10551-010-0434-0

Delafrooz, N., Taleghani, M., \& Nouri, B. (2014). Effect of green marketing on consumer purchase behavior. QScience Connect, 5(10), 1-9. https://doi.org/10.5339/connect.2014.5

D'Souza, C., Taghian, M., Lamb, P., \& Peretiakos, R. (2006). Green products and corporate strategy: an empirical investigation. Society and Business Review, 1(2), 144-157. https://doi.org/10.1108/17465680610669825

Farias, S. A., \& Aguiar, E. C. (2015). Percepção da presença dos outros consumidores e sua relação com emoções e valor hedônico de compra. Revista de Administração de Empresas, 55(6), 712-723. http://dx.doi.org/10.1590/S0034-759020150609

Ferreira, M., \& Zambaldi, F. (2019). The Mediating Role of Consumer Engagement with the Brand Community and Its Effect on Corporate Reputation. International Journal on Media Management, 21(1), 45-64. https://doi.org/10.1080/14241277.2019.1585354

Goriparthi, R. K., \& Tallapaly, M. (2017). Consumers' Attitude in Green Purchasing. FIIB Business Review, 6(1), 34-44. https://doi.org/10.1177/2455265820170106

Gurtner, S., \& Soyez, K. (2016). How to catch the generation Y: Identifying consumers of ecological innovations among youngsters. Technological Forecasting \& Social Change, 106(11), 101-107. https://doi.org/10.1016/j.techfore.2016.02.015

Hartmann, P., \& Ibáñez, V. A. (2006). Green value added. Marketing Intelligence \& Planning, 24(7), 673680. https://doi.org/10.1108/02634500610711842

Hill, M. M., \& Hill, A. (2016). Investigação por Questionário (2nd ed.). Edições Sílabo.

Jang, Y. J., Kim, W. G., \& Bonn, M. A. (2011). Generation Y consumers' selection attributes and behavioral intentions concerning green restaurants. International Journal of Hospitality Management, 30(4), 803-811. https://doi.org/10.1016/j.ijhm.2010.12.012

Joshi, Y., \& Rahman, Z. (2016). Predictors of young consumer's green purchase behaviour. Management of Environmental Quality: An International Journal, 27(4), 452-472. https://doi.org/10.1108/MEQ-05-2015-0091

Júnior, J. C. de O., Silva, A. W. P., Veiga Neto, A. R., Castro, A. B. C. \& Lima, D. S. V. R. (2020). Determining factors of environmental concern in purchasing decisions. REMark-Revista Brasileira de Marketing, 19(4), 888-918. https://doi.org/10.5585/remark.v19i4.16470

Juwaheer, T. D., Pudaruth, S., \& Noyaux, M. E. (2012). Analysing the impact of green marketing strategies on consumer purchasing patterns in Mauritius. World Journal of Entrepreneurship, Management and Sustainable Development, 8(1), 36-59. https://doi.org/10.1108/20425961211221615

Kanchanapibul, M., Lacka, E., Wang, X., \& Chan, H. K. (2014). An empirical investigation of green purchase behaviour among the young generation. Journal of Cleaner Production, 66(1), 528-536. https://doi.org/10.1016/j.jclepro.2013.10.062

Kianpour, K., Jusoh, A., \& Asghari, M. (2012). Importance of Price for Buying Environmentally Friendly Products. Journal of Economics and Behavioral Studies, 4(6), 371-375. ISSN: 2220-6140 
Kong, W., Harun, A., Sulong, R. S., \& Lily, J. (2014). The influence of consumers' perception of green products on green purchase intention. International Journal of Asian Social Science, 4(8), 924939. http://www.aessweb.com/pdf-files/ijass-2014-4(8)-924-939.pdf

Lee, K. (2008). Opportunities for green marketing: young consumers. Marketing Intelligence \& Planning, 26(6), 573-586. https://doi.org/10.1108/02634500810902839

Lima, E., Costa, C., \& Félix, G. (2019). Guilt and Pride Emotions and their Influence on the Intention of Purchasing Green Products. Revista Comportamento do Consumidor, 3(2), 70-84. https://doi.org/10.51359/2526-7884.2019.240028

Mangini, R., Amaral, L., Conejero, M., \& Pires, C. (2020). Greenwashing Study and Consumers' Behavioral Intentions. Revista Comportamento do Consumidor, 4(3), 229-244. https://doi.org/10.51359/2526-7884.2020.244488

Mitchell, M. A., McLean, P., \& Turner, G. B. (2005). Understanding Generation X... Boom or Bust Introduction. Business Forum, 26-30. https://search.proquest.com/docview/210199662?pq-origsite=gscholar\&fromopenview=true

Morton, L. P. (2003). Segmenting publics: Targeting Generation X. Public Relations Quarterly, 48(4), 4345.

Motta, S. L. (2008). Competitividade baseada no Marketing Ecológico. Revista de Ciências da Administração, 10(22), 128-145. https://doi.org/10.5007/2175-8069.2008v10n22p128

Noble, C. H., \& Mokwa, M. P. (1999). Implementing marketing strategies: developing and testing a managerial theory. Journal of Marketing, 63(4), 57-73. https://doi.org/10.2307/1251974

Ordun, G. (2015). Millennial (Gen Y) Consumer Behavior, Their Shopping Preferences and Perceptual Maps Associated With Brand Loyalty. Canadian Social Science, 11(4), 40-55. https://doi.org/10.3968/6697

Papadas, K.-K., Avlonitis, G. J., \& Carrigan, M. (2017). Green Marketing orientation: Conceptualization, scale development and validation. Journal of Business Research, 80, 236-246. https://doi.org/10.1016/j.jbusres.2017.05.024

Parment, A. (2013). Generation Y vs. Baby Boomers: Shopping behavior, buyer involvement and implications for retailing. Journal of Retailing and Consumer Services, 20(2), 189-199. https://doi.org/10.1016/j.jretconser.2012.12.001

Pestana, M. H., \& Gageiro, J. N. (2014). Análise de Dados para Ciências Sociais: A Complementaridade do SPSS (6 ed.). Sílabo.

Pickett-Baker, J., \& Ozaki, R. (2008). Pro-environmental products: marketing influence on consumer purchase decision. Journal of Consumer Marketing, 25(5), 281-293. https://doi.org/10.1108/07363760810890516

Rahbar, E., \& Wahid, N. A. (2011). Investigation of green marketing tools' effect on consumers' $\begin{array}{lllll}\text { purchase behavior. Business Strategy } & \text { Series, }\end{array}$ https://doi.org/10.1108/17515631111114877

Rissanen, H., \& Luoma-Aho, V. (2016). (Un)willing to engage? First look at the engagement types of millennials. Corporate Communications: An International Journal, 21(4), 500-515. https://doi.org/10.1108/CCIJ-06-2015-0038

Roberts, J. A. (1996). Green Consumers in the 1990s: Profile and Implications for advertising. Journal of Business Research, 36(3), 217-231. https://doi.org/10.1016/0148-2963(95)00150-6

Shrum, L. J., McCarty, J. A., \& Lowrey, T. M. (Summer de 1995). Buyers Characteristics of Green Consumer and Their Implications for Advertising Strategy. Journal of Advertising, XXIV(2), 71-82. https://doi.org/10.1080/00913367.1995.10673477

Sousa, J. M., Castro, A. L. O., \& Oliveira, J. M. S. R. (2016). Marketing Verde: A influência dos produtos ecológicos na decisão de compra dos consumidores nos supermercados de uma cidade no interior de Minas Gerais. Revista Conexão Ciência, 11(2), 71-82, https://periodicos.uniformg.edu.br:21011/periodicos/index.php/testeconexaociencia/article/v iewFile/446/499

Straughan, R. D., \& Roberts, J. A. (1999). Environmental segmentation alternatives: a look at green consumer behavior in the new millennium. Journal of Consumer Marketing, 16(6), 558-575. https://doi.org/10.1108/07363769910297506 
Toaldo, A, M. M. \& Luce, F. B. (2006). Estratégia de marketing: contribuições para a teoria em marketing. Revista de Administração de Empresas, 46(4), 25-35. https://doi.org/10.1590/S003475902006000400004

Williams, K. C., \& Page, R. A. (2011). Marketing to the Generations. Journal of Behavioral Studies in Business, 3(1), 37-53. https://www.researchgate.net/publication/242760064 